\title{
A Correlative Approach for Identifying Complex Phases by Electron Backscatter Diffraction and Transmission Electron Microscopy
}

\author{
Seon-Hyeong $\mathrm{Na}^{1}$, Jae-Bok Seol ${ }^{2}$, Majid Jafari ${ }^{1}$, Chan-Gyung Park ${ }^{1,2, *}$ \\ ${ }^{1}$ Department of Materials Science and Engineering, Pohang University of Science and Technology, Pohang 37673, Korea \\ ${ }^{2}$ National Institute for Nanomaterials Technology, Pohang University of Science and Technology, Pohang 37673, Korea
}

*Correspondence to:

Park CG,

Tel: +82-54-279-2139

Fax: +82-54-279-0249

E-mail: cgpark@postech.ac.kr

Received January 9, 2017

Revised February 17, 2017

Accepted February 18, 2017
A new method was introduced to distinguish the ferrite, bainite and martensite in transformation induced plasticity (TRIP) steel by using electron backscatter diffraction (EBSD) and transmission electron microscopy (TEM). EBSD is a very powerful microstructure analysis technique at the length scales ranging from tens of nanometers to millimeters. However, iron BCC phases such as ferrite, bainite and martensite cannot be easily distinguished by EBSD due to their similar surface morphology and crystallographic structure. Among the various EBSD-based methodology, image quality (IQ) values, which present the perfection of a crystal lattice, was used to distinguish the iron BCC phases. IQ values are very useful tools to discern the iron BCC phases because of their different density of crystal defect and lattice distortion. However, there are still remaining problems that make the separation of bainite and martensite difficult. For instance, these phases have very similar IQ values in many cases, especially in deformed region; therefore, even though the IQ value was used, it has been difficult to distinguish the bainite and martensite. For more precise separation of bainite and martensite, IQ threshold values were determined by a correlative TEM analysis. By determining the threshold values, iron BCC phases were successfully separated.

Key Words: Transformation induced plasticity steel, Electron backscatter diffraction, Transmission electron microscopy

\section{INTRODUCTION}

The most important issue for understanding the overall properties of the complicated phases steels is to clearly identify the all phases in microstructure. A typical example for complicated phases steels is transformation induced plasticity (TRIP) steels (De Cooman, 2004; Fischer et al., 2000), which contain austenite, ferrite, bainite, martensite, carbide, etc. The quantity of each phase has a decisive effect on the mechanical properties, but it's not easy to quantify. In order to distinguish the complicated phases, several methods have been proposed such as X-ray diffraction (XRD), color tint etching and light optical microscopy (LOM) and electron backscattering diffraction (EBSD) based on scanning electron microscopy (SEM). XRD provides the sufficiently accurate information about the volume fraction of each phase by calculating the intensity of diffraction peaks. However, it is the average information from a large area or volume. So, it is difficult to recognize the exact location of each phase and specific information about the local area. LOM based on color tint etching is very easy to characterize the microstructure of complex, multi-phases steels. However, color tint etching does not always show the precise difference because it depends only on the local carbon contents. Moreover, LOM has a relatively low resolution to observe the details of the fine microstructure such as TRIP steels containing sub-micrometer sized grains.

(a) This is an open-access article distributed under the terms of the Creative Commons Attribution Non-Commercial License (http://creativecommons.org/licenses/by-nc/4.0) which permits unrestricted noncommercial use, distribution, and reproduction in any medium, provided the original work is properly cited.

Copyrights @ 2017 by Korean Society of Microscopy 
EBSD is very sensitive to surface condition and needs to sufficient operation time but can provide quite accurate information on the crystal structure, phase distribution, etc. inside the specific local area.

In conventional TRIP steels, EBSD is a very useful technique to separate the retained austenite FCC phase from the other BCC phases since FCC and BCC phases have clearly different Kikuchi diffraction pattern (Herrera et al., 2011; Petrov et al., 2007; Zaefferer et al., 2004). However, separation of iron BCC phases is relatively difficult because they have similar crystallographic structure. Regarding that martensite has an almost the same crystallographic structure as BCC, low Kikuchi diffraction pattern quality and density of particular grain boundaries or dislocations are utilized to separate it from ferrite. A typical example is dual phase steel (Calcagnotto et al., 2008; Pinard et al., 2013). Both phases have been successfully distinguished because they show clearly different features. However, in TRIP steel, identifying the martensite is more difficult because of the existence of bainite structure, which indicates very similar characteristics to martensite in terms of morphology, crystallographic structure, carbon contents and dislocation density, etc. Therefore, traditional method has limitation for separating bainite and martensite in the steels having complex microstructure such as TRIP steel.

Over the past decade, many studies have benefited from the various tools of EBSD to distinguish the bainite and martensite. One of them is image quality (IQ), the quality metrics of the acquired electron back-scattering Kikuchi pattern that are derived from Hough transformation (Petrov et al., 2007; Wilson \& Spanos, 2001; Wu et al., 2005). IQ describes the intensity of the Hough peaks which is affected by elastically distorted lattices and density of crystalline defects or residual stresses (Humphreys, 2001). Normally, martensite has a very low IQ and confidence index (CI) since martensite contains a larger amount of lattice defects than bainite and ferrite. IQ can be a key to solving the question for the separation of bainite and martensite. However, the challenges are still remained; that is, in most cases, the IQ value of bainite is too close to that of martensite making their separation problematic. The IQ values of bainite and martensite are partially merged in IQ distribution. Therefore, effective separation of bainite and martensite IQ values in IQ distribution has been a challenging issue. Mujica et al. (2010) have performed the EBSD-IQ tool in conjunction with optical microscopy to characterize TRIP and TWIP steels joined by laser welding. IQ value allowed the quantification of ferritic, martensitic, and bainitic fractions produced by different combinations of laser welding and induction heating. In the base metal, BCC phases were easily separated since they show obviously distinct peaks in IQ distribution. On the other hand, in heat affected zone (HAZ), three different phases were completely merged as if there is a single phase. The author used the same threshold value as base metal in HAZ to separate ferrite, bainite and martensite. However, it may cause errors because IQ value is sensitive to either surface condition or contamination. Base metal and HAZ are completely different regions. It cannot be expected that these two regions, which may have different optimal etching condition, exhibit the same surface condition. So, care must be taken when using the same threshold value in different area. A more important problem is that we cannot verify the results only by using EBSD. In order to solve these problems, we need to apply a new method when IQ values of BCC phases are completely merged. Focused ion beam (FIB) and transmission electron microscopy (TEM) were applied to observe the detailed structure of each phase in the region of interest. By using FIB and TEM, we could find a more reasonable threshold value when bainite and martensite merge into one peak in IQ distribution and verify the EBSD phase identification results.

\section{MATERIALS AND METHODS}

The starting material in this study is low carbon TRIP steel with a typical heat treatment procedure explained elsewhere (Matsumura et al., 1987a, 1987b, 1991). Austenitizing, which means the pearlite and ferrite transformation to austenite, occurs during heating and soaking up to intercritical annealing temperature, and the subsequent cooling triggers ferrite formation. The following holding at the bainitic transformation temperature promotes the austenite to bainite phase transformation, while parts of the austenite transforms to martensite during the last stages of cooling process. Hence, the final structure of TRIP steel contains FCC phases such as retained austenite and BCC phases such as ferrite, bainite and martensite. Carbon content $(0.1 \mathrm{wt} \%)$ is slightly lower than commercial TRIP steel leading to a lower fraction of retained austenite. The sample was machined from the center of its width along the rolling direction-normal direction, and mechanical polishing was performed using $3 \mu, 1 \mu, 0.25$ $\mu \mathrm{m}$ diamond suspension. An area of $0.5 \mathrm{~cm}^{2}$ was electrochemically polished on the Struers Lectropol-5 using an electrolyte of $10 \%$ perchloric acid/methanol solution at $60 \mathrm{~V}$ and room temperature for $10 \mathrm{~s}$. SEM was carried out by using FIB, FEI Helios 600. The EBSD information was obtained using an EDAX-TSL EBSD system operating at $20 \mathrm{kV}$ accelerating voltage and $22 \mathrm{nA}$ probe current. The analysis area was 1,672 $\mu \mathrm{m}^{2}$ scanned with the step size of $0.5 \mu \mathrm{m}$ in a hexagonal grid at 3,500× magnification. Only ferrite (BCC) and austenite (FCC) crystallographic data was used from TSL database.

The data were further processed in TSL-OIM software (version 7, EDAX, USA). A CI value of more than 0.1 was used for phase identification to raise the confidence of the results. And tolerance angle of 15 degrees was applied for grain identi- 
fication. The "neighbor phase correlation" and "grain dilation" clean up algorithm was applied to the orientation data after their acquisition. Bright-field (BF) and dark-field (DF) TEM images as well as selected area diffraction patterns were obtained with a JEOL 2100F microscope (JEOL Ltd., Japan) operated at $200 \mathrm{kV}$. All images were post-processed using the Gatan Digital Micrograph (DM). In order to make correlative approach of TEM and EBSD, FIB (FEI Helios 600) lift out method (Giannuzzia \& Stevieb, 1999) was employed.

\section{RESULTS AND DISCUSSION}

Fig. 1 shows the microstructure of TRIP steel as observed by SEM after electro-chemical polishing with $10 \%$ perchloric acid. Due to its flat surface, ferrite phase is clearly distinguished from the other phases. Ferrite has a quick and regular etching speed during the electro-chemical polishing because of its smoothness and low carbon content. The retained austenite, bainite and martensite are also visible in SEM images. The fraction of austenite is very small, while bainite and martensite have largely occupied the most parts owing to their low nominal carbon content. The martensite phase partly exhibits an evident difference with other phases since it has a clear lath structure. However, in most cases, it is impossible to entirely distinguish martensite from bainite based on SEM observation.

The definitive method to distinguish the crystal structure of each phase over a large area is EBSD Kikuchi pattern technique. The EBSD is very useful to distinguish the BCC and FCC phase. As illustrated in Fig. 2, austenite is easily separated from BCC phases due to the clearly different

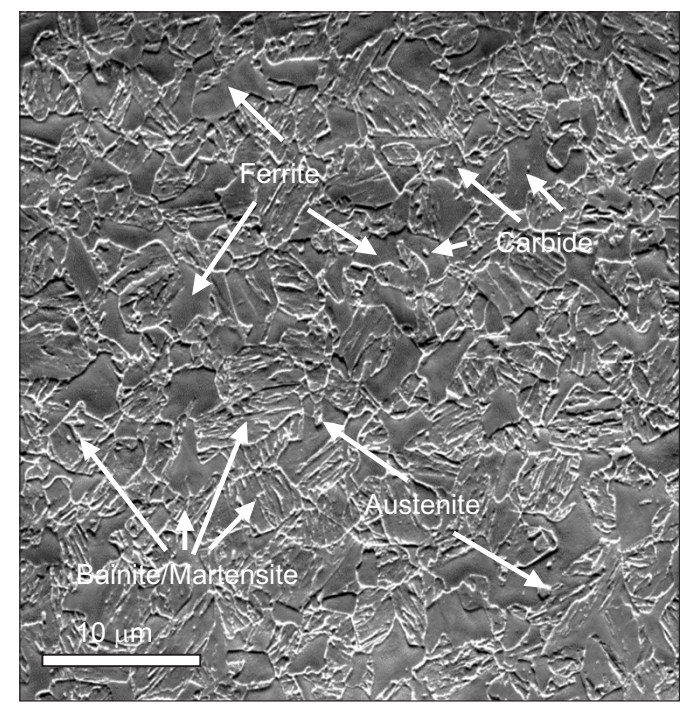

Fig. 1. Scanning electron microscopy micrograph of transformation induced plasticity steel after electro-chemical polishing showing retained austenite, ferrite, bainite, martensite and carbides precipitate. crystallographic structure. Generally, TRIP steel should be carefully handled during preparation for EBSD because the retrained austenite is susceptible to mechanical stress, especially in the case of 0.1C TRIP steel. In this study, a smaller fraction of the retained austenite $(3 \%)$ was detected as compared to conventional TRIP steels. This may be due to the stress induced martensitic transformation of the retained austenite during the mechanical polishing. The XRD analysis, performed to obtain the correct information about the retained austenite volume fraction, showed 5.0\% austenite for the TRIP steel, which is slightly higher than EBSD result (3\%). In the phase map, fundamentally separation of the bainite and martensite is impossible since the Kikuchi diffraction patterns of these phases are almost the same. On the other hand, IQ distribution could reveal the difference between bainite and martensite because of the different quality in Kikuchi pattern, but not in the shape. Martensite has higher lattice distortion than bainite; therefore, martensite exhibits a more blurred pattern image. In this study, the normalized grain average IQ value was adapted to reduce the grain boundary effect. Variations within the individual grains are lost, but the ability to compare the grains can be enhanced. Fig. $2 \mathrm{C}$ shows that all grains have different IQ value indicating the different lattice perfection; hence, each grain is easily separated not like the phase map. Each color points to the level of lattice distortion so that the closer color to blue signifies the higher lattice distortion. Although each grain has a unique value, the grains
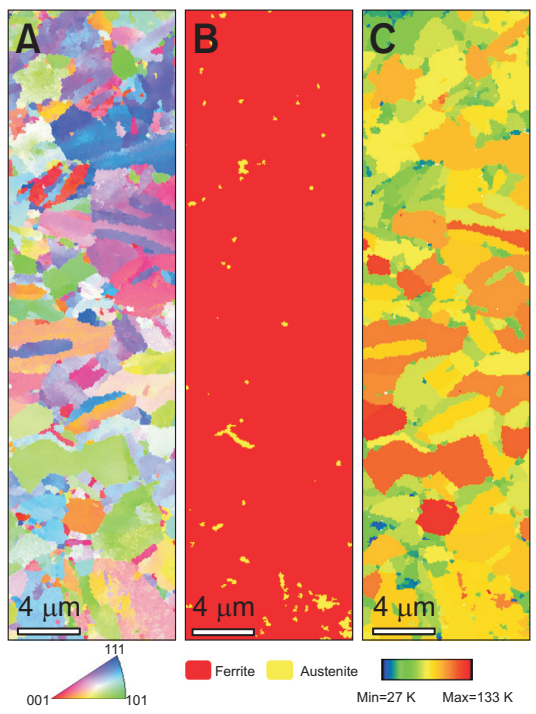

Fig. 2. Comparison of the electron backscatter diffraction maps of transformation induced plasticity steel. (A) Inversed pole figure map showing crystal orientations. (B) Phase map showing crystal structure; ferrite (red), austenite (yellow). (C) Average image quality (IQ) map. Inversed pole figure map shows the grain boundaries but identifying each grains is impossible. In phase map, all BCC phases are looks like same phases. On the other hand, average IQ map indicate that each grains can be separated according to degree of lattice distortion. 
are difficult to be sorted into three-groups, ie. ferrite, bainite and martensite, because their IQ values were very close to each other (Fig. 3). Threshold values of ferrite, bainite and martensite are essential for separation when each distribution is indistinguishable. In Fig. 3, the grain average IQ value distribution is merged into a single peak because bainite and martensite have too close Gaussian distribution. Therefore, the most important issue is to find a threshold value by using additional method since intuitionally separation of BCC phases in IQ distribution is very difficult.

A threshold value for separating ferrite from other phases is easy to be set because ferrite is easily divided from the other phases in SEM image with electro-chemical polishing as noted previously. The EBSD-IQ map was obtained in the same region of SEM observation. Ferrite has a very high IQ since it maintains the perfection of BCC crystal lattice. The map of ferrite grains can be roughly drawn by clipping the ferrite region in the grain average IQ distribution. Fig. 4 shows the ferrite maps extracted from the IQ map (Fig. 2) by selecting the slightly different threshold value. Red color region indicates the ferrite phase, but white color region is unknown. The ferrite volume fraction gradually increases as the threshold value for separating ferrite from bainite is gradually deceased. To find the exact fraction of ferrite, the threshold should be adjusted until the ferrite fraction and morphology remain unchanged in IQ map and SEM image. Fig. 4C demonstrates the ferrite phase in IQ map when a threshold value of 0.6168 was chosen. The red color region (ferrite) shows a very similar morphology to the SEM image; thus, it can be concluded that the phases with IQ value of higher than 0.6168 are ferrite with a high probability.

The threshold value of ferrite is relatively easy to find because of its simple morphology. On the other hand, finding the threshold values for bainite and martensite is very difficult

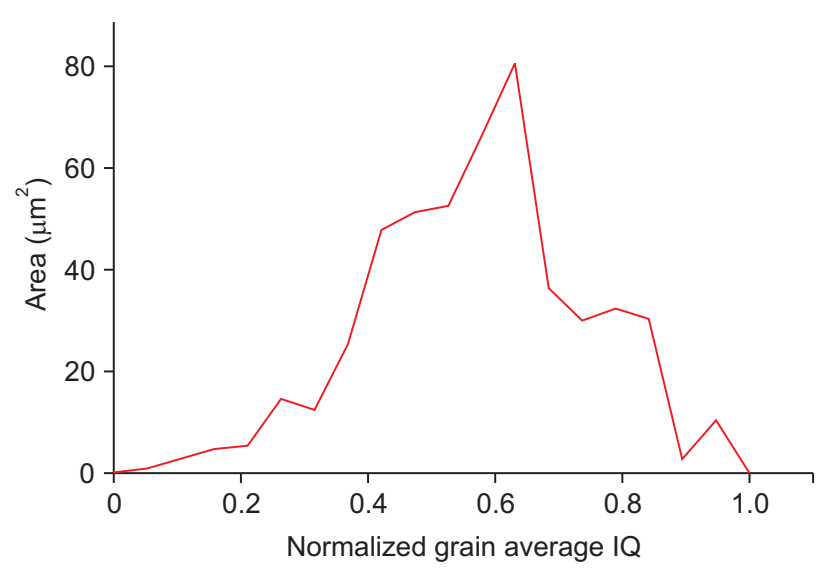

Fig. 3. Normalized grain-average image quality (IQ) distribution of iron BCC phases. Separation of BCC phases is impossible without correct threshold value. in comparison to ferrite because these phases have very similar morphology and crystallographic structure. It is impossible to distinguish the bainite and martensite by using SEM or EBSD phase map. Therefore, TEM was employed to observe the detailed morphology and micro-structure of bainite and martensite. In order to study the specific grains by using TEM, FIB was used for sampling. After the EBSD analysis, specific grains, which were unknown to be bainite or martensite, were chosen to prepare TEM samples by using lift-out method (Giannuzzia \& Stevieb, 1999). Sequences of the TEM sampling is as follows: First, an unknown grain which was very difficult to identify was chosen in SEM image. Then, EBSD information was obtained in the same region as SEM image. Subsequently, the average IQ distribution and individual average IQ value of each grain were obtained. Fig. 5A and B illustrate the SEM image of unknown grains (red line) and average IQ map in the same region. In Fig. $5 \mathrm{~B}$, the grain had a value of 0.0544 , which is very lower than the value in IQ distribution. This implies that this grain could be martensite with a high possibility since the lowest IQ value corresponds to highest lattice distortion. By using $\mathrm{Ga}+$ ion beam, upper and lower regions of this grain were removed, the process which is called trench milling. Fig. 5C shows the cross-sectional ion beam image of the target grain after trench milling. From this process, the exact location of the target grain can be verified before lift-out. After the lift-out, ion beam milling process was performed at $20 \mathrm{kV}$ for thinning and, finally, the ion beam damaged region was removed at $5 \mathrm{kV}$. Thickness of the sample was less than 100
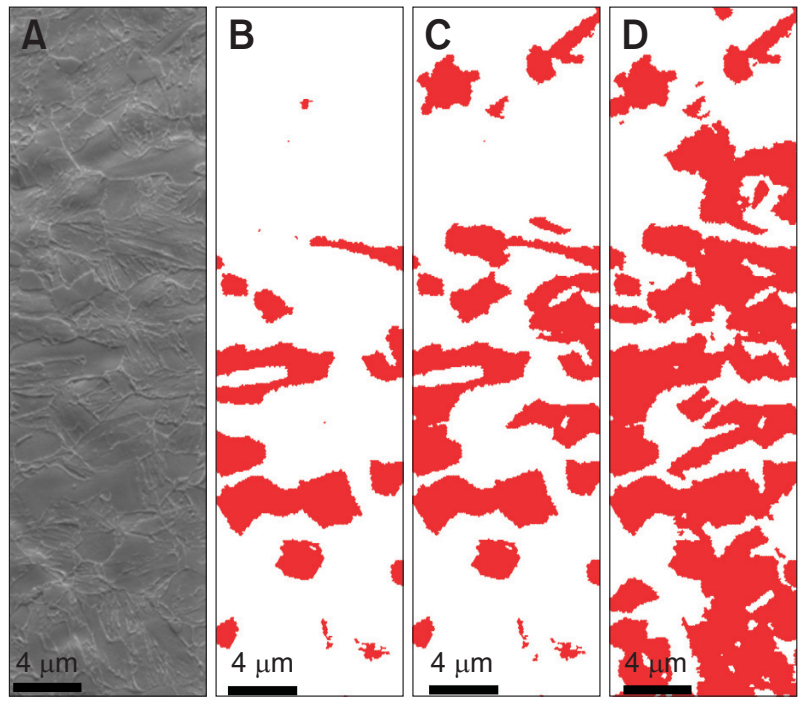

Fig. 4. Scanning electron microscopy micrograph showing microstructure of transformation induced plasticity steel and corresponding grainaverage image quality maps showing ferrite regions according to different threshold value; (B) 0.7776, (C) 0.6704, (D) 0.5732. (C) shows the most similar image with (A). 

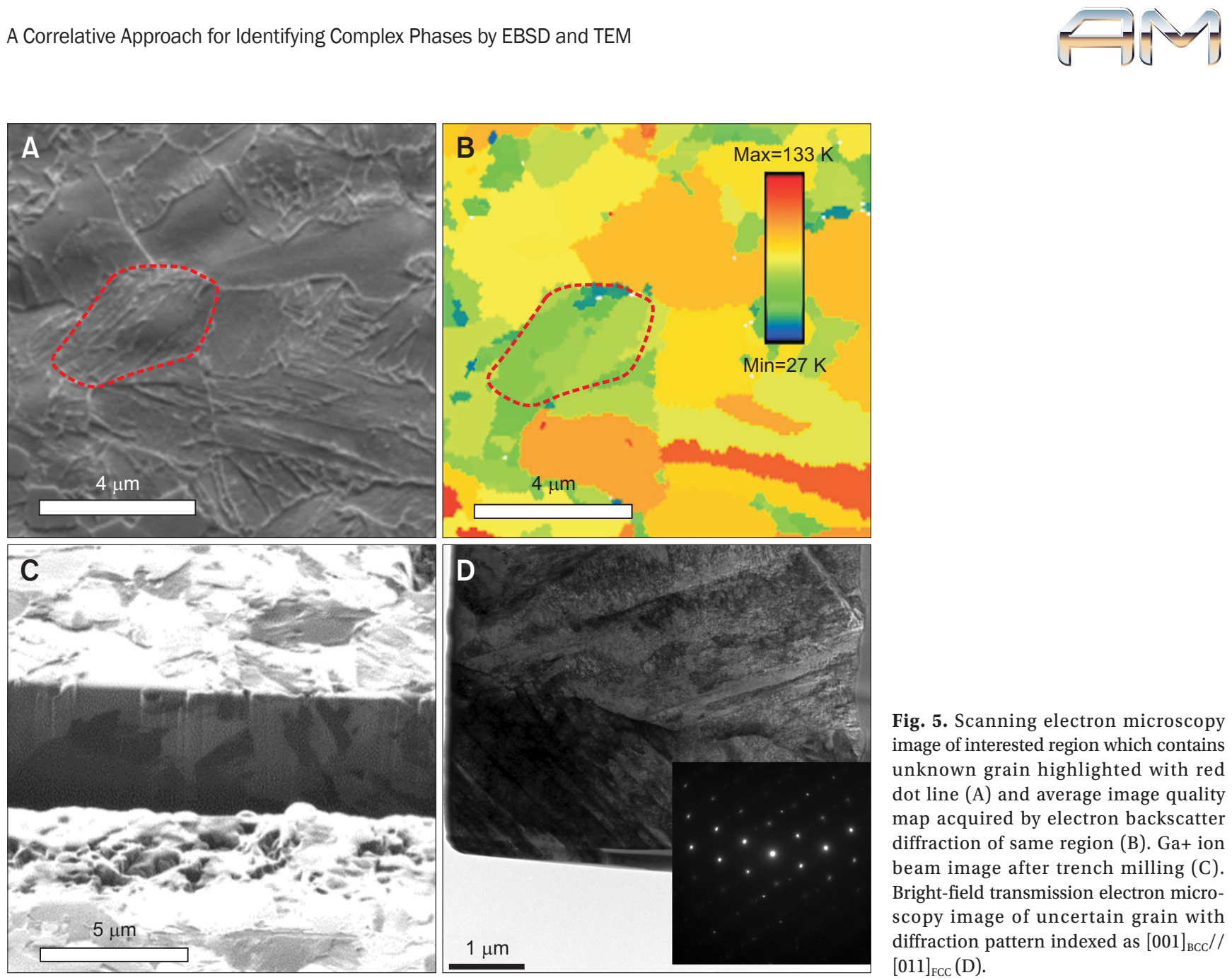

Fig. 5. Scanning electron microscopy image of interested region which contains unknown grain highlighted with red dot line (A) and average image quality map acquired by electron backscatter diffraction of same region (B). Ga+ ion beam image after trench milling $(C)$. Bright-field transmission electron microscopy image of uncertain grain with diffraction pattern indexed as $[001]_{\mathrm{BCC}} / /$ $[011]_{\mathrm{FCC}}(\mathrm{D})$.

$\mathrm{nm}$ after the final milling. From the BF TEM image, the detailed structure of fine grains can be clearly observed. Fig. 5 reveals the lath type structure and the corresponding diffraction pattern. Diffraction pattern directly shows the Baker-Nutting orientation relationship with the retained austenite and BCC structure. Overall, this unknown grain is lath martensite which has IQ value of 0.0544 . From this result, it can be inferred that the grains with IQ value of lower than 0.0544 represent lath martensite. However, IQ value of lath martensite also has variation as plotted in IQ distribution. In order to raise the accuracy, it is necessary to repeat the analysis at around 0.0544 by the same way.

The next step is to find the IQ value for bainite in IQ distribution. The threshold value to distinguish bainite and ferrite is already found. In order to identify the bainite region in IQ distribution, threshold value for separating bainite and martensite is needed. It is a natural idea that bainite is located just below the ferrite region and above the 0.6168 value in IQ value distribution. In order to determine the threshold value for bainite, the analysis should be repeated until bainite was found by the same method. As shown in Fig. 6, another unknown grains were chosen in different area. Grains have higher IQ value than lath martensite (0.0544) in Fig. 5 and lower than ferrite (0.6168). Fig. 6A and B depict the SEM image and IQ map of this unknown grain which is difficult to identify whether it is bainite or martensite, but it has higher IQ value than lath martensite. Similar to the previous method, FIB was used to make TEM sample of this unknown grain. Fig. 6C shows the cross-sectional image of the selected grain and corresponding diffraction pattern. The DF TEM image of the unknown grain in Fig. 6D is distinguished from the lath martensite image in Fig. 5. No carbide is observed and the retained austenite is located at the interface of granular shape BCC phases. Diffraction pattern could not show any kind of orientation relationship. Based on these evidences, one can conclude that the granular type grain is bainite. In the conventional TRIP steel, depending on the heat treatment, carbide free bainite can be included. Same experiment was repeated three times around $0.45 \sim 0.46$ value. Results show that 0.456 is the most suitable value for threshold. From this result, it can be inferred that most of bainite will show higher IQ value than 0.456 . From now on, we can distinguish the bainite, martensite and ferrite in the same sample by using only IQ value. Fig. 7 is a result of phase identification. 


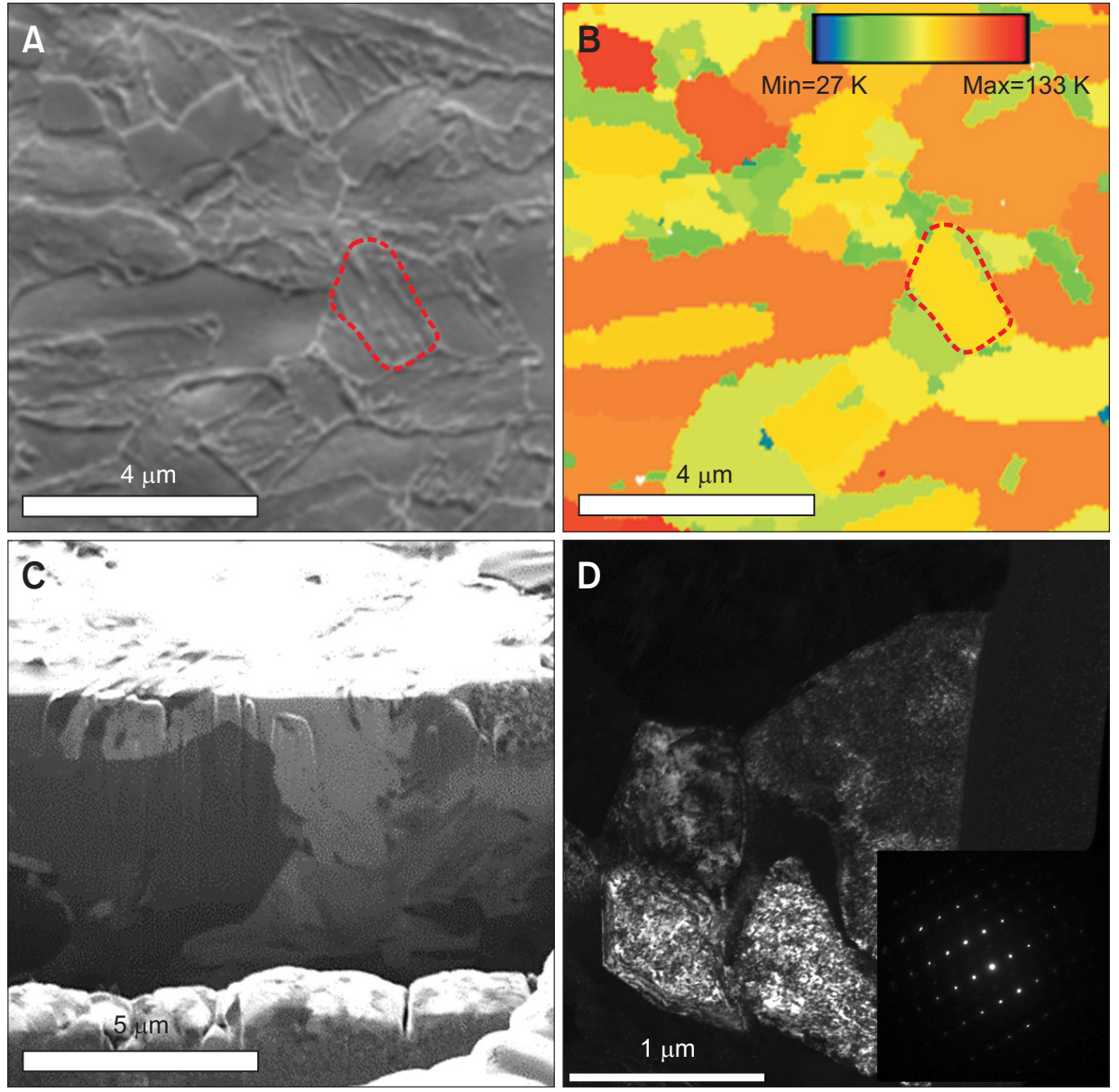

Fig. 6. Scanning electron microscopy image of interested region which contains unknown grain highlighted with red dot line (A) and average image quality map acquired by electron backscatter diffraction of same region (B). Ga+ ion beam image after trench milling (C). BrightfieldBF transmission electron microscopy image of unknown grain with diffraction pattern indexed as $[-113]_{\mathrm{BCC}}$ zone axis (D).

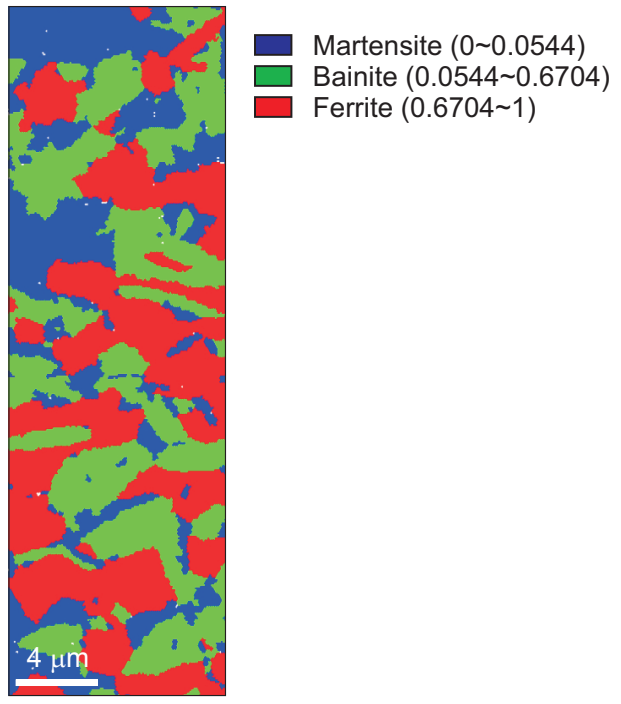

Fig. 7. Result of phase identification by using average image quality map. Blue, green and red color indicate martensite, bainite and ferrite phases. Austenite is ignored due to very small fraction.
The ferrite, bainite and martensite can be separated in IQ distribution because the IQ value of each phase is already known. From this result, a new IQ map, which indicates the fraction and exact location of each phase, can be plotted.

\section{CONCLUSIONS}

In this study, we attempted to solve the problem of determination of the volume fraction and location of BCC phases in conventional TRIP steel by using IQ distribution. Ferrite is relatively easy to separate from the other phases because of its distinguishing morphology in SEM after electro-chemical polishing. However, bainite and martensite are not separated on the SEM and EBSD phase map since both phases have similar surface morphology and crystallographic structure. A separation based on the IQ value is a reasonable way since IQ represents the lattice distortion, and martensite differs from bainite in terms of lattice distortion. IQ has limitation to separate when bainite and martensite have a merged single peak. The threshold values are very essential when IQ is utilized for phase separation. As a result, a new approach was introduced to find the threshold value for bainite and 
martensite. 1. EBSD analysis was performed in a certain area which should have clean surface. Ferrite was easily separated from the other phases in SEM and EBSD average IQ map because of its distinguishing morphology in SEM. Bainite and martensite are very difficult to distinguish in SEM image; therefore, TEM sampling was carried out by lift-out method using FIB. 2. Unknown grains, which are difficult to identify whether they are bainite or martensite, were chosen for a detailed structural examination. By using FIB lift-out method, TEM samples were prepared to observe the cross-section of the chosen grains. TEM images showed a detailed morphology such as retained austenite along the lath boundary and crystallographic information about the fine particles. 3. From the TEM images and diffraction pattern, the origin of the phases could be determined. By repeating this process, we could find the reasonable threshold value for separation. Once the threshold value is detected for ferrite, bainite and martensite, these phases are easily separated only by using IQ value. 4 . Threshold value could be changed depending on the sample because IQ value is very sensitive to the surface condition. We should find the threshold value again in different sample or etching condition. Nevertheless, this method is very useful to distinguish bainite and martensite in micro-scale. By applying this method, vast information about volume fraction and location of bainite and martensite can be quickly obtained.

\section{CONFLICT OF INTEREST}

No potential conflict of interest relevant to this article was reported.

\section{REFERENCES}

Calcagnotto M, Ponge D, and Raabe D (2008) Ultrafine grained ferrite/ martensite dual phase steel fabricated by large strain warm deformation and subsequent intercritical annealing. ISIJ Int. 48, 1096-1101.

De Cooman B C (2004) Structure-properties relationship in TRIP steels containing carbide-free bainite. Curr. Opin. Solid State Mater. Sci. 8, 285-303.

Fischer F D, Reisner G, Werner E, Tanaka K, Cailletaud G, and Antretter T (2000) A new view on transformation induced plasticity (TRIP). Int. J. Plast. 16, 723-748.

Giannuzzia L A and Stevieb F A (1999) A review of focused ion beam milling techniques for TEM specimen preparation. Micron 30, 197204.

Herrera C, Ponge D, and Raabe D (2011) Design of a novel Mn-based 1 GPa duplex stainless TRIP steel with $60 \%$ ductility by a reduction of austenite stability. Acta Mater. 59, 4653-4664.

Humphreys F J (2001) Grain and subgrain characterisation by electron backscatter diffraction. J. Mater. Sci. 36, 3833-3854.

Matsumura O, Sakuma Y, and Takechi H (1987a) Enhancement of elongation by retained austenite in intercritical annealed $0.4 \mathrm{C}-1.5 \mathrm{Si}-$ 0.8Mn Steel. ISIJ Int. 27, 570-579.

Matsumura O, Sakuma Y, and Takechi H (1987b) TRIP and its kinetics aspects in austempered $0.4 \mathrm{C}-1.5 \mathrm{Si}-0.8 \mathrm{Mn}$ steel. Script Metall. 21 , 1301-1306.
Matsumura O, Sakuma Y, and Takechi H (1991) Mechanical properties and retained Austenite in intercritically heat treated bainite-transformed steel and their variations with $\mathrm{Si}$ and $\mathrm{Mn}$ additions. Metall. Trans. A 22A, 489-498.

Mujica L, Weber S, Pinto H, Thomye C, and Vollertsene F (2010) Microstructure and mechanical properties of laser-welded joints of TWIP and TRIP steels. Mater. Sci. Eng. A 527, 2071-2078.

Petrov R, Kestens L, Wasilkowska A, and Houbaert Y (2007) Microstructure and texture of a lightly deformed TRIP-assisted steel characterized by means of the EBSD technique. Mater. Sci. Eng. A 447, 285-297.

Pinard P T, Schwedt A, Ramazani A, Prahl U, and Richter S (2013) Characterization of dual-phase steel microstructure by combined submicrometer EBSD and EPMA carbon measurements. Microsc. Microanal. 19, 996-1006.

Wilson A W and Spanos G (2001) Application of orientation imaging microscopy to study phase transformations in steels. Mater. Charact. 46, 407-418.

Wu J, Wray P, Garcia C, Hua M, and Deardo A (2005) Image quality analysis: a new method of characterizing microstructures. ISIJ Inter. 45, 254-262.

Zaefferer S, Ohlert J, and Bleck W (2004) A study of microstructure, transformation mechanisms and correlation between microstructure and mechanical properties of a low alloyed TRIP steel. Acta Mater. 52, 2765-2778. 